\title{
Non-Hypersplenism Causes of Peripheral Cytopenias in Patients with Cirrhotic Portal Hypertension: A Review
}

\author{
Yunfu Lv ${ }^{1,3}$, Wan Yee Lau ${ }^{1,2^{*}}$, Jie Deng ${ }^{1,3}$, YeJuan $\mathbf{L i}^{1,3}$ and Yonghong Dong ${ }^{4}$
}

${ }^{1}$ Department of General Surgery, Hainan Province People's Hospital, Haikou (570311), China

${ }^{2}$ Faculty of Medicine, The Chinese University of Hong Kong, Shatin, Hong Kong SAR, China

${ }^{3}$ Hainan University, Haikou, 570228, China

${ }^{4}$ Department of General Surgery, Shanxi Province People's Hospital, Taiyuan, (030001), China

*Corresponding author: Wan Yee Lau, Department of General Surgery, Hainan Province People's Hospital, Haikou (570311), China; E-mail: josephlau@cuhk.edu.hk

Received date: Mar 5, 2016; Accepted date: May 11, 2016; Published date: May 16, 2016

Copyright: (c) 2016 Lv Y, et al. This is an open-access article distributed under the terms of the Creative Commons Attribution License, which permits unrestricted use, distribution, and reproduction in any medium, provided the original author and source are credited.

\begin{abstract}
Hypersplenism and non-hypersplenism factors, either alone or in combination, can cause peripheral cytopenias in patients with cirrhotic portal hypertension. Although non-hypersplenism factors account for only a small proportion of patients, they do exist. When peripheral cytopenias do not improve, or fail to improve adquately, or even become worse after splenectomy in these patients, non-hypersplenism factors should be considered. This review aims to provide an overview of non-hypersplenism factors.
\end{abstract}

Keywords: Cirrhotic portal hypertension; Peripheral cytopenias; Etiology; Non-Hypersplenism factor

\section{Introduction}

Peripheral cytopenias refer to the lower-than-normal counts of circulating leukocytes, erythrocytes, and platelets. Leukopenia can lead to decreased immunity with consequent susceptibility to infection. The main function of erythrocytes is to transport oxygen $\left(\mathrm{O}_{2}\right)$ and participate in carbon dioxide $\left(\mathrm{CO}_{2}\right)$ exhalation [1], and transportation of $\mathrm{O}_{2}$ by erythrocytes is achieved via hemoglobin $(\mathrm{Hb})$ within cells. The gas transportation function may be lost in extreme erythropenia or erythrocytic rupture resulting in escape of $\mathrm{Hb}$, tissue ischemia and hypoxia. Thrombocytopenia can lead to bleeding diathesis [2]. Theoretically and clinically, severe cytopenias affect prognosis [3], and can even lead to death [4]. Peripheral cytopenias can be caused by both hypersplenism and non-hypersplenism factors. These factors, either alone or in combination, can cause peripheral cytopenias in patients with cirrhotic portal hypertension. Peripheral cytopenias were previously thought to be caused by hypersplenism alone in patients with cirrhotic portal hypertension [5-7], and decreased blood cells levels would return to normal after splenectomy. However, in some patients, peripheral cytopenias do not improve, or fail adequately to improve, or become even worse after splenectomy. In such cases, nonhypersplenism factors should be considered. Unfortunately, little attention has been paid to non-hypersplenism factors as they are uncommon, and sometimes they coexist with hyperplenism factors. There have only been a few studies conducted on peripheral cytopenias caused by non-hyperplenism factors. This review aims to provide an overview on these non-hypersplenism factors (Table 1). Although this is not a systematic review, the authors tried to include as many articles published on this field as possible to avoid introducing personal biases into this review.

\begin{tabular}{|l|}
\hline Hypersplenism Causes \\
\hline Non-hypersplenism Causes \\
\hline Toxic effects of viruses on bone marrow \\
\hline Hepatitis B \\
\hline Hepatitis C \\
\hline Human parvovirus B19 \\
\hline Human cytomegalovirus \\
\hline Hepatic dysfunction \\
\hline Immunodeficiency \\
\hline Dystrophy - ? poor nutrition \\
\hline Drugs toxicity \\
\hline Circulating platelet destruction \\
\hline Blood loss \\
\hline
\end{tabular}

Table 1: Hypersplenism and non-hypersplenism causes of peripheral cytopenias in patients with portal hypertension.

\section{Non-hypersplenism Factors}

\section{Toxic effects of viruses on bone marrow}

The bone marrow is a hematopoietic organ. Viral infection can lead to impaired cellular and humoral immunity, impaired ability to clear the virus, and consequent viral persistence, thus resulting in bone marrow hematopoietic dysfunction $[8,9]$. Hepatitis $B$ and $C$ viruses can cause bone marrow suppression and affect the development of all nucleated bone marrow cells, leading to hypoplastic anemia and cytopenias. In these cases, survival is possible only with bone marrow 
transplant [10]. Djordjevic et al. [11] pointed out that thrombocytopenia in patients with splenomegaly due to portal hypertension had a variety of causes, and that one important cause was bone marrow suppression by hepatitis virus. Even with liver transplant, cytopenias can also be caused by continuous toxic effects of hepatitis virus on bone marrow [12]. Human parvovirus B19 and human cytomegalovirus (HCMV) also can produce inhibition of bone marrow. Espanol [13] believed that direct infection or abnormal immune mechanisms may explain thrombocytopenia in patients with chronic liver disease.

Forbes et al. [14] suggested that hepatic exogenous myofibroblasts played an important role in hepatic fibrosis in liver diseases of different etiologies. In hepatic fibrosis, bone marrow stem cells differentiate into hepatic endothelial parenchymal cells but not into myofibroblasts. This indicates that the change in hemopoietic function and inner environment of the bone marrow might be somehow related to, or interactive with, the occurrence and development of hepatic fibrosis or even hepatic cirrhosis. These findings suggest that bone marrow changes in patients with liver cirrhosis do not result from one single factor, but a combination of multiple factors, with a complicated regulatory mechanism. Their relationship and the detailed pathogenesis remain to be further investigated. Solving this puzzle will be of great importance to clinical treatment.

\section{Hepatic dysfunction}

The liver has many important physiological functions, such as detoxification, metabolism, bile secretion, and immune defense. Hepatic dysfunction can lead to dysfunction in detoxification, which results in accumulation of toxic substances in the body, thus affecting the bone marrow hematopoietic function. Sezai [15] found decreased serum albumin accompanied by significant thrombocytopenia in patients with cirrhotic portal hypertension. Liver disease complicated by thrombocytopenia occurs at an incidence of $15-70 \%$, and is usually mild to moderate in severity, which is considered to be a prognostic indicator.

Thrombopoietin (TPO) is almost exclusively produced by hepatocytes, and the number and functions of hepatocytes determine the production of TPO. In cirrhosis, there is a decrease in functional hepatocytes. A correlation between decreases in serum levels of TPO with platelet counts has been found by Wolber et al. [16] during transition from compensated to decompensated cirrhosis.

Many studies have pointed out that a deficiency of thrombopoietin (TPO) in liver disease was an important factor in the etiology of thrombocytopenia [17-19]. However, studies have also shown that there was no significant difference in TPO levels between thrombocytopenia and non-thrombocytopenia patients, suggesting that thrombocytopenia was unrelated to the TPO level [20], and may have been directly related to the splenic volume and platelet-associated immunoglobulin G (PAIgG) [21-23]. In addition, PAIgG can also bind to and destroy megakaryocytes and their precursors, thus inhibiting their differentiation and platelet formation [24], resulting in decreased production of blood cells.

Cirrhotic patients with a low hepatic functional reserve have low blood viscosity, low hematocrit, low platelet count, and prolong clotting time. Patients with low blood viscosity are at a high risk of bleeding [25]. During cirrhosis, there is a reduction in reverse T3 5'deiodinase activity, serum total and free T3, and even in T4 in severe cases. These factors affect the hematopoietic system, thus resulting in decreased production of blood cells. Hypoxemia is likely to occur in severe cirrhosis, which can also cause peripheral cytopenias.

Metabolism disorders and abnormal membrane lipid synthesis of erythrocytes can result in increased erythrocyte morphology fragility, leading to shortened life and increased destruction of erythrocytes [25].

\section{Immunodeficiency}

Immunodeficiency is a low resistance to infection caused by developmental defects in the immune system or immune response dysfunction, with clinical manifestations of recurrent infections or serious infectious diseases.

Due to immune dysfunction or disorders, a variety of blood cell autoantibodies are produced in cirrhotic patients and blood cells are easily ingested by phagocytes, leading to cytopenias [26-28]. Liver disease complicated by thrombocytopenia is most closely related with cirrhosis [14], and is also significantly related with antiplatelet autoantibodies. Olariu [19] believed that bone marrow suppression, immune deficiency, and liver fibrosis were determinants of thrombocytopenia.

Thrombocytopenia due to immunodeficiency can be autoimmune or acquired immune thrombocytopenia. The former is more common [26] and includes cytopenias caused by post-autoimmune hepatitis cirrhosis and autoimmune lymphoproliferative syndrome $[29,30]$. The latter, also known as basic immune thrombocytopenia, is an acquired immune disease common in chronic lymphocytic leukemia [31,32], variable immunodeficiency disease $[33,34]$, and hemophagocytic syndrome [35], presenting with abnormal bleeding due to decreased platelet counts. Thrombocytopenia due to immunodeficiency occurs in cases of increased antibody-mediated platelet destruction coupled with decompensation due to bone marrow defect [36]. It was previously reported that the average life of platelets in immune thrombocytopenia was 12 hours, which was shorter than that in hypersplenism (56 hours) and bone marrow hypoplasia (102 hours) [37]. These suggest severe destruction of platelets by immunodeficiency.

\section{Dystrophy}

It has long been considered that poor nutrition leads to liver cirrhosis, but there is still a lack of evidence to support this view. In animal experiments, food deficient in protein, choline and vitamin results in changes in the liver. Unfortunately, these changes do not have the secondary changes in blood vessels typical of a cirrhotic liver in man, and the changes are usually reversible if a diet rich in protein is given to the animal. Furthermore, mild fibrous hyperplasia happens in only a small number of these animals. Patients with liver disease often have malabsorption of nutrients, which leads to malnutrition. Lavigne et al. [38] found vitamin deficiency was a common factor of pancytopenia. Patients with vitamin deficiency present with severe iron deficiency anemia and thrombocytopenia [39], and have a mean corpuscular volume higher than patients with erythropenia caused by other factors.

\section{Drugs toxicity}

Allergic reactions and drugs toxicity also affect liver function, leading to cytopenias related to iron storage proteins. Improvement in liver function with an immediate increase in serum ferritin have been observed after discontinuation of treatment [40]. Thus, serum ferritin 
levels may be a reliable indicator in the diagnosis of liver dysfunction and cytopenias. 5-Fluorouracil is an anticancer drug, whose toxicity can lead to cytopenias and diarrhea [41,42]. Most other anti-cancer drugs, such as cyclophosphamide and oxaliplatin, also have such side effects $[43,44]$. Some antibiotics, such as $\beta$-lactam-containing antibiotics, can induce cytopenias in patients with hepatic decompensation, which may be due to the abnormal liver metabolism caused by excessive $\beta$-lactam-containing antibiotics, leading to suppression of leukocyte precursor cells in the bone marrow [45].

\section{Circulating platelet destruction}

Platelets are commonly found in human blood. Binding of human anti-platelet autoantibodies to receptors (glycoproteins) on the platelet membrane in any part of the body leads to increased platelet destruction $[46,47]$. Destruction of platelets may occur in the spleen or in the circulating blood. The destruction of circulating platelets remains even after splenectomy, leading to a low level of platelets. If platelets account for a large proportion of cells in the spleen, peripheral platelet count rises soon after splenectomy; otherwise, peripheral platelet count does not change much after splenectomy, and may even be lower than before surgery. Cho et al. [48] found that destructed peripheral platelets became immature platelet fragments (IPF). IPF\% is used as a reference value in trials, and increased IPF\% has become an important indicator for laboratory diagnosis of thrombocytopenia. Any causes in hematopoietic dysfunction can lead to liver damage and liver functional abnormalities, aggravating liver fibrosis and cirrhosis.

\section{Blood loss}

Srichaikul [49] suggested that gastrointestinal bleeding can cause pancytopenia. The volume of blood loss is related to peripheral cytopenias. A small volume of blood loss does not lead to peripheral cytopenias, whereas a large volume of blood loss may result in peripheral cytopenias, in addition to severe anemia [50]. Patients with portal hypertension are often complicated by gastrointestinal bleeding, thus leading to cytopenias [51]. Patients with renal transplantation are susceptible to gastrointestinal tract cytomegalovirus infection, presenting with gastrointestinal bleeding, and significant cytopenia can occur soon after bleeding [52].

Peripheral cytopenias due to non-hypersplenism factors are complicated, since a variety of non-hypersplenism factors are often combined, and they often coexist with hypersplenism factors.

\section{Treatment}

Different treatments should be administered for peripheral cytopenias due to non-hypersplenism factors according to the specific cause.

\section{Etiological treatment}

Antiviral treatment should be administered for cytopenias caused by viral infection, and combination of pegylated interferon and ribavirin has been shown to be a feasible treatment [53]. Vitamin supplements should be given for pancytopenia caused by vitamin deficiency [54]. Medication should be discontinued immediately for cytopenias caused by excessive use of anti-cancer drugs or certain antibiotics.

\section{Symptomatic treatment}

Supplements should be given for what is deficient, for example, transfusion of erythrocytes for erythropenia, platelets for thrombocytopenia, whole blood for leukopenia, and coagulation factors for coagulation factor deficiency. For autoimmune cytopenia, special treatment should be given with high-dose corticosteroids and intravenous immune globulin. Eltrombopag is a new, orally active thrombopoietin receptor agonist that can stimulate platelet production and increase platelet counts in thrombocytopenia due to HCV-related cirrhosis [38]. Continuous BKT140 monotherapy has been shown to significantly increase the levels of megakaryocytes and hematopoietic progenitor cells in bone marrow, thus reducing the severity of thrombocytopenia before and after chemotherapy, and shortening the duration of thrombocytopenia [41]. BKT140 is a chemokine receptor (CXCR4) antagonist.

\section{Liver transplantation}

Liver transplantation can be considered for patients with severe liver cirrhosis and severe cytopenias. Liver transplantation not only greatly improves long-term survival in patients with decompensated cirrhosis, but also is an effective treatment for peripheral cytopenias $[55,56] \mathrm{Li}$ [57] and Chu [58] demonstrated that liver transplantation combined with concurrent splenectomy did not increase the risk in the treatment of cirrhosis and hypersplenism, and suggested it may provide better effects.

\section{Acknowledgements}

This work was supported by the special fund for international cooperation projects from the Science and Technology Foundation of Hainan Province, China (Grant No. KJHZ2015-28).

\section{References}

1. Doan CA (1949) Hypersplenism. Bulletin of the New York Academy of Medicine 25: 625-650.

2. Wu CC, Cheng SB, Ho WM (2004) Appraisal of concomitant splenectomy in liver resection for hepatocellular carcinoma in cirrhotic patients with hypersplenic thrombocytopenia. Surgery 136: 660-668.

3. Lv Y, Han X, Gong X (2015) Grading of Peripheral Cytopenias Caused by Nonalcoholic Cirrhotic Portal Hypertension and Its Clinical Significance. Cell biochemistry and biophysics 71: 1141-1145.

4. Yoshida I, Yoshino T, Takeuchi M (2005) Elderly patient with varicellazoster virus-associated hemophagocytic syndrome refractory to steroid therapy. Rinsho ketsueki The Japanese journal of clinical hematology 46: 1229-1232.

5. Pereira J, Accatino L, Alfaro J, Brahm J, Hidalgo P, et al. (1995) Platelet autoantibodies in patients with chronic liver disease. American journal of hematology 50: 173-178.

6. Jiang A, Zhang S, Li Z (2011) miR-615-3p promotes the phagocytic capacity of splenic macrophages by targeting ligand-dependent nuclear receptor corepressor in cirrhosis-related portal hypertension. Experimental biology and medicine 236: 672-680.

7. Zucker ML, Hagedorn CH, Murphy CA, Stanley S, Reid KJ, et al. (2012) Mechanism of thrombocytopenia in chronic hepatitis $\mathrm{C}$ as evaluated by the immature platelet fraction. International journal of laboratory hematology 34: 525-532.

8. Brown KE, Tisdale J, Barrett AJ, Dunbar CE, Young NS (1997) Hepatitisassociated aplastic anemia. The New England journal of medicine 336: 1059-1064.

9. Cudillo L (2009) Aplastica anemia and viral hepatitis. Mediterranean journal of hematology and infectious diseases 1: e2009026. 
10. Young NS, Maciejewski J (1997) The pathophysiology of acquired aplastic anemia. The New England journal of medicine 336: 1365-1372.

11. Djordjevic J, Svorcan P, Vrinic D, Dapcevic B (2010) Splenomegaly and thrombocytopenia in patients with liver cirrhosis. Vojnosanitetski pregled Military-medical and pharmaceutical review 67: 166-169.

12. Nakamae H, Storer B, Sandmaier BM (2011) Cytopenias after day 28 in allogeneic hematopoietic cell transplantation: impact of recipient/donor factors, transplant conditions and myelotoxic drugs. Haematologica 96: 1838-1845.

13. Espanol I, Gallego A, Enriquez J (2000) Thrombocytopenia associated with liver cirrhosis and hepatitis $\mathrm{C}$ viral infection: role of thrombopoietin. Hepato-gastroenterology 47: 1404-1406.

14. Forbes SJ, Russo FP, Rey V (2004) A significant proportion of myofibroblasts are of bone marrow origin in human liver fibrosis. Gastroenterology 126: 955-963.

15. Sezai S, Kamisaka K, Ikegami F (1998) Regulation of hepatic thrombopoietin production by portal hemodynamics in liver cirrhosis. The American journal of gastroenterology 93: 80-82.

16. Wolber EM, Ganschow R, Burdelski M, Jelkmann W (1999) Hepatic thrombopoietin mRNA levels in acute and chronic liver failure of childhood. Hepatology 29: 1739-1742.

17. Tomikawa M, Akahoshi T, Sugimachi K (2010) Laparoscopic splenectomy may be a superior supportive intervention for cirrhotic patients with hypersplenism. Journal of gastroenterology and hepatology 25: 397-402.

18. Peck-Radosavljevic M, Wichlas M, Zacherl J (2000) Thrombopoietin induces rapid resolution of thrombocytopenia after orthotopic liver transplantation through increased platelet production. Blood 95: 795-801.

19. Olariu M, Olariu C, Olteanu D (2010) Thrombocytopenia in chronic hepatitis C. Journal of gastrointestinal and liver diseases : JGLD 19: 381-385.

20. Panasiuk A, Prokopowicz D, Zak J, Panasiuk B (2004) Reticulated platelets as a marker of megakaryopoiesis in liver cirrhosis; relation to thrombopoietin and hepatocyte growth factor serum concentration. Hepato-gastroenterology 51: 1124-1128.

21. Rios R, Sangro B, Herrero I, Quiroga J, Prieto J (2005) The role of thrombopoietin in the thrombocytopenia of patients with liver cirrhosis. The American journal of gastroenterology 100: 1311-1316.

22. Sanjo A, Satoi J, Ohnishi A, Maruno J, Fukata M, et al. (2003) Role of elevated platelet-associated immunoglobulin $\mathrm{G}$ and hypersplenism in thrombocytopenia of chronic liver diseases. Journal of gastroenterology and hepatology 18: 638-644.

23. Liu TT, Wong WJ, Hou MC, Lin HC, Chang FY, et al. (2006) Hemorheology in patients with liver cirrhosis: special emphasis on its relation to severity of esophageal variceal bleeding. Journal of gastroenterology and hepatology 21: 908-913.

24. Lv YF, Li XQ, Gong XG, Xie XH, Han XY, et al. (2013) Effect of surgery treatment on hypersplenism caused by cirrhotic portal hypertension. Minerva Chir 68: 409-413.

25. Jiao YF, Okumiya T, Saibara T, Kudo Y, Sugiura T (2001) Erythrocyte creatine as a marker of excessive erythrocyte destruction due to hypersplenism in patients with liver cirrhosis. Clinical biochemistry 34 : 395-398.

26. Zheng P, Chang X, Lu Q, Liu Y (2013) Cytopenia and autoimmune diseases: a vicious cycle fueled by mTOR dysregulation in hematopoietic stem cells. Journal of autoimmunity 41: 182-187.

27. Barzaghi F, Passerini L, Bacchetta R (2012) Immune dysregulation, polyendocrinopathy, enteropathy, $\mathrm{x}$-linked syndrome: a paradigm of immunodeficiency with autoimmunity. Frontiers in immunology 3:211.

28. Friedman LS (1999) The risk of surgery in patients with liver disease. Hepatology 29: 1617-1623.

29. Voinchet H, Etienne G, Ghiringelli CB (2010) Splenic marginal zone lymphoma and autoimmunity: report of six cases. La Revue de medecine interne / fondee par la Societe nationale francaise de medecine interne 31: 4-11.
30. Visco C, Cortelezzi A, Moretta F (2014) Autoimmune cytopenias in chronic lymphocytic leukemia at disease presentation in the modern treatment era: is stage $\mathrm{C}$ always stage C? Leukemia \& lymphoma 55: 1261-1265.

31. Tandra P, Krishnamurthy J, Bhatt VR, Newman K, Armitage JO, et al. Autoimmune cytopenias in chronic lymphocytic leukemia, facts and myths. Mediterranean journal of hematology and infectious diseases 5: e2013068.

32. Oksenhendler E, Fieschi C (2007) Common variable immunodeficiency. La Revue du praticien 57: 1687-1690.

33. Haymore BR, Mikita CP, Tsokos GC (2008) Common variable immune deficiency (CVID) presenting as an autoimmune disease: role of memory B cells. Autoimmunity reviews 7: 309-312.

34. John MJ, Rajasekhar R, Mathews V (2008) Autoimmune lymphoproliferative syndrome (ALPS): a rare cause of immune cytopenia. Indian pediatrics 45: 148-150.

35. Nogueira MV, Vidal L, Terra B, Pagot T, Salluh JI, et al. (2009) Hemophagocytic syndrome associated with cytomegalovirus infection in a severely immunocompromised AIDS patient: case report. Braz J Infect Dis 13: 72-73.

36. Mingot Castellano ME (2010) Romiplostim: an advance in the treatment of idiopathic thrombocytopenic purpura. Methods and findings in experimental and clinical pharmacology 32: 39-43.

37. Richards JD, Thompson DS (1979) Assessment of thrombocytopenic patients for splenectomy. Journal of clinical pathology 32: 1248-1252.

38. Lavigne C, Lavigne E, Massenet D, Binet C, Bremond JL, et al. (2005) Role of vitamin deficiency in pancytopenia in Djibouti, Findings in a series of 81 consectutive patients. Medecine tropicale : revue du Corps de sante colonial 65: 59-63.

39. Abrishami F, Golshan A (2013) Frequency of iron deficiency anemia in girls studying in mashhad high schools. Iranian journal of pediatric hematology and oncology 3: 143-148.

40. Miyazaki M, Tanaka M, Ueda A (2011) Acute liver failure caused by drug-induced hypersensitivity syndrome associated with hyperferritinemia. World journal of gastroenterology : WJG 17: 4928-4931.

41. Abraham M, Weiss ID, Wald H (2013) Sequential administration of the high affinity CXCR4 antagonist BKT140 promotes megakaryopoiesis and platelet production. British journal of haematology 163: 248-259.

42. Saif MW, Lee AM, Offer SM, McConnell K, Relias V, et al. (2014) A DPYD variant (Y186C) specific to individuals of African descent in a patient with life-threatening 5-FU toxic effects: potential for an individualized medicine approach. Mayo Clinic proceedings 89: 131-136.

43. Salem ML, Al-Khami AA, El-Nagaar SA (2012) Kinetics of rebounding of lymphoid and myeloid cells in mouse peripheral blood, spleen and bone marrow after treatment with cyclophosphamide. Cellular immunology 276: 67-74.

44. Cobo F, De Celis G, Pereira A, Latorre X, Pujadas J, et al. (2007) Oxaliplatin-induced immune hemolytic anemia: a case report and review of the literature. Anti-cancer drugs 18: 973-976.

45. Singh N, Yu VL, Mieles LA, Wagener MM (1993) Beta-Lactam antibioticinduced leukopenia in severe hepatic dysfunction: risk factors and implications for dosing in patients with liver disease. The American journal of medicine $94: 251-256$.

46. Ziakas PD, Routsias JG, Giannouli S, Tasidou A, Tzioufas AG, et al. (2006) Suspects in the tale of lupus-associated thrombocytopenia. Clinical and experimental immunology 145: 71-80.

47. van den Bemt PM, Meyboom RH, Egberts AC (2004) Drug-induced immune thrombocytopenia. Drug safety : an international journal of medical toxicology and drug experience 27: 1243-1252.

48. Cho YG, Lee JH, Kim DS, Lee HS, Choi SI (2007) Clinical usefulness of the simple technique to diagnose thrombocytopenia using immature platelet fraction. The Korean journal of laboratory medicine 27: 1-6.

49. Srichaikul T, Punyagupta S, Kanchanapoom T, Chanokovat C, Likittanasombat K, et al. (2008) Hemophagocytic syndrome in Dengue 
Citation: Lv Y, Lau WY, Deng J, Li JY, Dong Y (2016) Non-Hypersplenism Causes of Peripheral Cytopenias in Patients with Cirrhotic Portal Hypertension: A Review. J Hypertens 5: 223. doi:10.4172/2167-1095.1000223

Page 5 of 5

hemorrhagic fever with severe multiorgan complications. Journal of the Medical Association of Thailand Chotmaihet thangphaet 91: 104-109.

50. Kankirawatana S, Kupatawintu P, Juji T (2001) Neonatal alloimmune thrombocytopenia due to anti-Nak(a). Transfusion 41:375-377.

51. Leite LA, Domingues AL, Lopes EP (2013) Relationship between splenomegaly and hematologic findings in patients with hepatosplenic schistosomiasis. Revista brasileira de hematologia e hemoterapia 35: 332-336.

52. Ardalan M (2012) Rare presentations of cytomegalovirus infection in renal allograft recipients. Nephro-urology monthly 4: 431-436.

53. McHutchison JG, Dusheiko G, Shiffman ML (2007) Eltrombopag for thrombocytopenia in patients with cirrhosis associated with hepatitis C. New England Journal of Medicine 357: 2227-2236.

54. Iacobellis A, Cozzolongo R, Minerva N (2014) Feasibility of pegylated interferon and ribavirin in hepatitis C-related cirrhosis with neutropenia or thrombocytopenia. Digestive and liver disease : official journal of the
Italian Society of Gastroenterology and the Italian Association for the Study of the Liver 46: 621-624.

55. Tsukahara A, Sato Y, Yamamoto S (2003) Thrombopoietin levels and peripheral platelet counts following living related donor liver transplantation. Hepato-gastroenterology 50: 227-230.

56. O'Leary JG, Yachimski PS, Friedman LS (2009) Surgery in the patient with liver disease. Clinics in liver disease 13: 211-231.

57. Li DW, Du CY, Fan B, Huang P, Luo SQ, et al. (2012) Impact of simultaneous splenectomy and orthotopic liver transplantation in patients with end-stage liver diseases and splenic hyperfunction. Hepatobiliary \& pancreatic diseases international : HBPD INT 11: 489-493.

58. Chu HC, Hsieh CB, Hsu KF, Fan HL, Hsieh TY, et al. (2015) Simultaneous splenectomy during liver transplantation augments antiviral therapy in patients infected with hepatitis $\mathrm{C}$ virus. American journal of surgery 209: 180-186. 